\title{
La incertidumbre en los tiempos del COVID-19
}

\author{
Uncertainty in the time of COVID-19
}

\author{
A incerteza nos tempos do COVID-19
}

\author{
Diana Cárdenas, MD, $\mathrm{PhD}^{1}$
}

https://doi.org/10.35454/rncm.v4n1.254

La pandemia COVID-19, sin precedentes, presenta desafíos para los gobiernos, los sistemas de salud y la sociedad en todo el mundo. Organizaciones, sociedades científicas y expertos internacionales han destacado la centralidad de la atención nutricional en el tratamiento de COVID-19. Se ha informado sobre un amplio espectro de oportunidades donde la terapia nutricional podría jugar un papel importante en el curso de la enfermedad. Además, se ha enfatizado acerca de la importancia de mantener un estilo de vida y una dieta saludables durante la cuarentena ${ }^{(1)}$.

Hoy, el mundo se enfrenta a la pandemia del SARSCoV-2 con una "doble carga" de malnutrición, por lo que un porcentaje de la población mundial podría tener una forma grave de COVID-19 asociada a la desnutrición y otro porcentaje a la obesidad. Una publicación liderada por el doctor Juan B. Ochoa, muestra como en términos generales, existen tres fenotipos de riesgo diferentes: 1. el paciente adulto mayor frágil, 2. el paciente con enfermedad crónica y 3. el paciente con obesidad grave y mórbida. Estos tres fenotipos representan diferentes riesgos nutricionales y necesitan diversas intervenciones nutricionales $^{(2)}$. Por lo tanto, el desafío de la pandemia del SARS-CoV-2 no es solo proteger a los adultos mayores y a los pacientes polimórbidos, sino también a aquellos afectados por la desnutrición y la obesidad.

Las sociedades de nutrición han publicado sus propias guías de manejo con base en la opinión de expertos y en

Facultad de Medicina, Instituto de Investigación en Nutrición, Genética y Metabolismo, Universidad El Bosque, Bogotá, D.C, Colombia. dianacardenasbraz@gmail.com la experiencia del manejo del COVID en los países que primero hicieron frente al virus (Italia, China). Entre los expertos existe un consenso sobre los beneficios de la terapia nutricional ${ }^{(3-7)}$ (Tabla 1). Por ejemplo, los expertos italianos en Lombardía, el centro de la crisis de COVID-19 en Italia, recomiendan de manera relevante el uso de suplementos nutricionales tempranos para pacientes no críticamente enfermos hospitalizados por COVID-19 para combatir la inflamación severa y la anorexia ${ }^{(3)}$. Las directrices colombianas acentúan la identificación de pacientes con riesgo nutricional para iniciar una atención nutricional óptima y oportuna en el marco de los derechos humanos. Esto último es particularmente importante porque la población colombiana ha estado expuesta a conflictos civiles durante décadas, y la reciente migración forzada de venezolanos a Colombia sin duda agrava la situación ${ }^{(4)}$.

Los expertos también concuerdan al afirmar que los pacientes con COVID-19 que presentan complicaciones respiratorias agudas y requieren hospitalización en la UCI están en riesgo importante de desnutrición. Las perturbaciones metabólicas de estos pacientes conducen a la pérdida de masa y función del músculo esquelético (sarcopenia), lo que a su vez podría conducir a mayor morbilidad, mala calidad de vida y discapacidad, incluso tiempo después del alta de la $\mathrm{UCI}^{(5)}$. Por lo tanto, las recomendaciones de los expertos se centran en alcanzar los objetivos de energía y proteína, aún si se indica nutrición enteral a través de sonda nasogástrica o nasoduodenal, incluso durante la posición decúbito prono. Estas recomendaciones deben considerarse en el escenario clínico actual caracterizado por una escasez importante de ventiladores y otros recursos, de médicos 
Tabla 1. Ejemplos de guías y recomendaciones internacionales sobre nutrición en el paciente con COVID-19

\begin{tabular}{|c|c|}
\hline $\begin{array}{l}\text { World Health Organization } \\
\text { - Consejería nutricional para adultos durante la pandemia }\end{array}$ & $\begin{array}{l}\text { http://www.emro.who.int/nutrition/ } \\
\text { nutrition-infocus/nutrition-advice-for- } \\
\text { adults-during-the-covid-19-outbreak.html }\end{array}$ \\
\hline $\begin{array}{l}\text { Academy of Nutrition and Dietetics } \\
\text { - Recursos relacionados con la nutrición para personas y familias durante las } \\
\text { condiciones desafiantes de la pandemia }\end{array}$ & https://www.eatright.org/coronavirus \\
\hline $\begin{array}{l}\text { American Society for Parenteral and Enteral Nutrition } \\
\text { - Terapia nutricional en pacientes con COVID -19 que requieren cuidados en la UCI } \\
\text { - Nutrición e hidratación: armas clave en la lucha contra COVID-19 }\end{array}$ & $\begin{array}{l}\text { https://www.nutritioncare.org/Guidelines_ } \\
\text { and_Clinical_Resources/Resources_for_ } \\
\text { Clinicians_Caring_for_Patients_with__ } \\
\text { Coronavirus/ }\end{array}$ \\
\hline $\begin{array}{l}\text { American Society for Nutrition } \\
\text { - Hacer de la salud y la nutrición una prioridad durante la pandemia }\end{array}$ & https://www.nutricare.org/COVID19/ \\
\hline $\begin{array}{l}\text { British Association for Parenteral and Enteral Nutrition } \\
\text { - Declaraciones y directrices para la terapia nutricional en pacientes que requieren } \\
\text { NIV y CPAP durante el COVID-19 }\end{array}$ & $\begin{array}{l}\text { https://www.bapen.org.uk/resources- } \\
\text { and-education/education-and-guidance/ } \\
\text { covid-19 }\end{array}$ \\
\hline $\begin{array}{l}\text { The Brazilian Association for Parenteral and Enteral Nutrition and The Brazilian Intensive } \\
\text { Care Medicine Association } \\
\text { - Declaración nutricional para hacer frente a COVID-19 en pacientes hospitalizados }\end{array}$ & $\begin{array}{l}\text { https://www.braspen.org/post/parecer- } \\
\text { braspen-amib }\end{array}$ \\
\hline $\begin{array}{l}\text { Asociación Colombiana de Nutrición Clínica } \\
\text { - Recomendaciones nutricionales para pacientes hospitalizados con infección por } \\
\text { SARS-CoV-2 }\end{array}$ & $\begin{array}{l}\text { https://revistanutricionclinicametabolismo. } \\
\text { org/index.php/ } \\
\text { nutricionclinicametabolismo }\end{array}$ \\
\hline $\begin{array}{l}\text { The European Society for Clinical Nutrition and Metabolism } \\
\text { - Declaraciones de expertos de ESPEN y orientación práctica para el manejo } \\
\text { nutricional de personas con infección por SARS-CoV-2 }\end{array}$ & https://www.clinicalnutritionjournal.com/ \\
\hline $\begin{array}{l}\text { La Academia Española de Nutrición y Dietética } \\
\text { - Recomendaciones alimentarias y nutricionales para la población española en la } \\
\text { crisis sanitaria de covid-19 }\end{array}$ & $\begin{array}{l}\text { https://www.academianutricionydietetica. } \\
\text { org/noticia.php?id=113 }\end{array}$ \\
\hline $\begin{array}{l}\text { Sociedad Francófona de Nutrición Clínica y Metabolismo } \\
\text { - Opinión de expertos sobre la estrategia de manejo nutricional hospitalario }\end{array}$ & $\begin{array}{l}\text { https://www.sfncm.org/images/stories/ } \\
\text { ARTICLES/2020/PEC_NUT_HOPITAL_ } \\
\text { COVID19_SFNCM.pdf }\end{array}$ \\
\hline $\begin{array}{l}\text { Equipo multidisciplinario de expertos, Lombardia, Italia } \\
\text { - Suplementación nutricional precoz en pacientes no críticamente enfermos } \\
\text { hospitalizados por la nueva enfermedad por coronavirus de } 2019 \text { (COVID-19): } \\
\text { justificación y viabilidad de un protocolo pragmático compartido }\end{array}$ & $\begin{array}{l}\text { https://www.sciencedirect.com/science/ } \\
\text { article/pii/S0899900720301180 }\end{array}$ \\
\hline
\end{tabular}

y personal de salud en general, una carga de trabajo diaria creciente y un alto riesgo de transmisión nosocomial.

Desde mi punto de vista, el principal reto se encuentra en la toma de decisiones sobre el manejo del COVID19 en un escenario caracterizado por la incertidumbre. Según la Real Academia de la Lengua la incertidumbre se define como "la falta de certidumbre, de certeza". La certeza es "el conocimiento seguro y claro de algo; la firme adhesión de la mente a algo conocible, sin temor de errar”. Es decir, se toman decisiones que conciernen la vida y la muerte de miles de personas enfermas sin un conocimiento sólido y el temor constante de causar daño. Las decisiones así tomadas ponen en juego los principios $y$ valores éticos de beneficencia, no maleficencia, justicia, equidad y respeto a la dignidad humana. Barrocas y colaboradores en un artículo publicado en Nutrition in Clinical Practice proponen, ante la incertidumbre sobre el curso de la pandemia de COVID-19, un marco ético para la toma de decisiones en un contexto de escasez de recursos y hacen énfasis en la importancia de los protocolos éticos y la toma de decisiones con enfoque multidisciplinario ${ }^{(8)}$. 
Frente a este escenario, se ha visto una afluencia preocupante de artículos científicos. Una búsqueda realizada en PUBMED, del 1 de enero al 15 de noviembre de 2020, muestra que se han publicado más de 60,000 artículos referentes al COVID-19 y SARS-CoV-2. Lograr esta cantidad de artículos en tan poco tiempo, significa que se han publicado de forma apresurada y algunas veces, evadiendo las exigencias de calidad editorial. Reflejo de esto es el no despreciable número de artículos retractados de prestigiosas revistas. Al mes de noviembre de 2020, se han retirado 38 artículos de manera definitiva y 3 de forma temporal ${ }^{(9)}$. Por ejemplo, se retractó un artículo que afirmaba de manera errónea que la suficiencia de vitamina $\mathrm{D}$ (25-hidroxivitamina $\mathrm{D}$ sérica de al menos $30 \mathrm{ng} / \mathrm{ml}$ ), reducía el riesgo de resultados clínicos adversos en pacientes con infección por COVID-19. Las retractaciones de artículos polémicos de las revistas The Lancet $y$ New England Journal of Medicine ponen en evidencia un serio problema de confianza en la investigación y alimenta la polémica sobre los intereses económicos y políticos en la ciencia. Sin embargo, si la publicación de estos artículos fracasó en su proceso editorial, con seguridad a nivel de revisión por pares, el escrutinio de la comunidad científica, probablemente impulsado por la atención mediática sobre la investigación del coronavirus, permitió detectar los errores en un tiempo récord. Estas retractaciones y las controversias generadas no son un caso excepcional. Según Ivan Oransky, cada año son retractados unos 1500 artículos por ausencia de solidez científica ${ }^{(9)}$. La crisis del COVID-19 dio visibilidad a este grave problema.

El manejo nutricional de los pacientes con COVID-19 no se escapa de este escenario. No existe certeza sobre el impacto metabólico del virus en el paciente y, por lo tanto, tampoco hay claridad sobre la mejor manera de abordar la problemática nutricional ni las necesidades nutricionales de los pacientes ventilados con COVID-19. Según la opinión de expertos, las necesidades nutricionales son similares a las de cualquier paciente con síndrome de dificultad respiratoria aguda (SDRA) ${ }^{(10)}$. En este contexto, no se ha visto una afluencia de artículos científicos, por el contrario, la escasez de artículos es inquietante. En una búsqueda en PUBMED se encontraron 1200 artículos sobre "COVID-19 and nutrition" y 766 artículos sobre "SARS-CoV-2 and nutrition" lo que equivale a 1,6\% y a 1,7\% del total de artículos respectivamente.

Sin lugar a dudas, no se trata de la cantidad de información sino de la calidad de la evidencia científica. Es decir, que para salir de la incertidumbre se requiere conocimiento que surja del método científico con los estándares de calidad y que su publicación cumpla el rigor editorial necesario. La opinión de los expertos es fundamental, pero debe ser refutada o confirmada bajo los estándares de la ciencia, con estudios aleatorizados y controlados.

La principal preocupación en el campo de la nutrición clínica es la escasez y la mala calidad de las investigaciones publicadas. Un ejemplo de esta aseveración y del peligro de la mala ciencia en nutrición es el artículo de Yu PJ, publicado en la revista Journal of Parenteral and Enteral Nutrition. Los autores señalan que los pacientes en estado crítico con COVID-19 se encuentran en un estado hipermetabólico extremo, luego de publicar un estudio con 7 pacientes críticos con COVID-19, donde se encontró una mediana de gasto energético en reposo (REE) de $4044 \mathrm{kcal}$ / día obtenido mediante calorimetría indirecta. Los autores concluyen afirmando que es necesario "potenciar mayores requerimientos nutricionales para dichos pacientes" ${ }^{(11)}$. Este artículo sorprende no solamente por los altos valores de REE, sino también por la pobre descripción metodológica y la falta de información de los casos estudiados lo que impide realizar un análisis acertado sobre los resultados. No sabremos si los altos valores de REE se obtuvieron por fallas técnicas en la medición, por situaciones clínicas concretas (cuadro de shunt pulmonar severo) o si realmente se trata del perfil metabólico de los pacientes con COVID-19. Este tipo de artículos generan más incertidumbre que certeza y ponen en riesgo la vida de los pacientes.

Estas preocupaciones son compartidas también por la doctora M. Isabel T.D Correia en su artículo Nutrition in times of Covid-19, how to trust the deluge of scientific information. La doctora resalta el alto número de artículos publicados sobre COVID-19 provenientes de países inicialmente afectados por la enfermedad y que en su mayoría son observaciones epidemiológicas o documentos de opinión que no cumplen los estándares de calidad de la investigación. Además, resalta que la terapia nutricional para pacientes con Covid-19 es un tema que no se aborda con frecuencia en los estudios publicados, a pesar de su máxima relevancia en el enfoque holístico de cualquier individuo ${ }^{(12)}$.

Así las cosas, comparto con la doctora Correia que "la mejor atención al paciente debe combinar el razonamiento clínico y el conocimiento científico. El diluvio masivo de publicaciones no necesariamente significa una buena calidad científica." 


\section{Agradecimientos}

Las charlas y el intercambio de comunicación con M. Isabel T.D Correia, Serrana Tihista, Juan B. Ochoa y Fernando Carrasco han motivado y enriquecido el tema de este editorial.

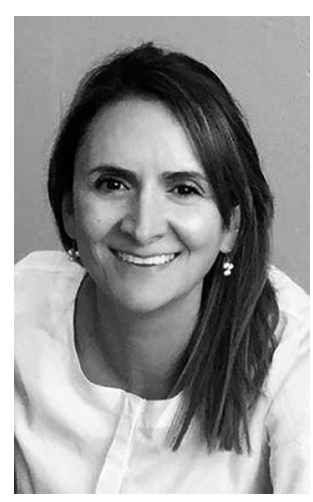

Diana Cárdenas, MD, PhD Editora de la Revista de Nutrición Clínica y Metabolismo, ACNC. Profesora e investigadora del Instituto de Nutrición, Genética y Metabolismo, Facultad de Medicina, Universidad El Bosque, Bogotá, Colombia.

\section{Referencias bibliográficas}

1. Muscogiuri G, Barrea L, Savastano S, Colao A. Nutritional recommendations for CoVID-19 quarantine. Eur J Clin Nutr. 2020; 74 : 850-1. https://doi.org/10.1038/s41430-020-0635-2

2. Ochoa JB, Cárdenas D, Goiburu ME, Bermúdez C, Carrasco F, Correia MITD. Lessons Learned in Nutrition Therapy in Patients With Severe COVID-19. JPEN J Parenter Enteral Nutr. 2020 24:10.1002/jpen.2005. doi: 10.1002/jpen.2005.

3. Caccialanza R, Laviano A, Lobascio F, Montagna E, Bruno R, Ludovisi S. Early nutritional supplementation in non-critically ill patients hospitalized for the 2019 novel coronavirus disease (COVID-19): Rationale and feasibility of a shared pragmatic protocol. Nutrition. 2020;71:110835. https://doi. org/10.1016/j.nut.2020.110835.

4. Bermúdez Ch, Pereira F, Trejos-Gallego D, Pérez A, Puentes M, LM López. Recomendaciones nutricionales de la
Asociación Colombiana de Nutrición Clínica para pacientes hospitalizados con infección por SARS-CoV-2. Rev Nutr Clín Metab. 2020;3(1):74-85 https://doi.org/10.35454/rncm. v3n1.066.

5. Barazzoni R, bischoff, Breda J, Wickramasinghe K, Krznaric Z, Nitzan D, Pirlich M, Singer P. ESPEN expert statements and practical guidance for nutritional management of individuals with SARS-CoV-2 infection Clin Nutr. 2020;39(6):1631-8. doi:10.1016/j.clnu.2020.03.022.

6. Martindale R, Patel J, Taylor B, Warren M, McClave S. Nutrition therapy in the patients with COVID -19 disease requiring ICU Care. [Internet] (consultado el 25 de Julio 2020) Disponible en: https://www.nutritioncare.org/ Guidelines_and_Clinical_Resources/Resources_for_ Clinicians_Caring_for_Patients_with_Coronavirus/.

7. Alhazzani W, Hylander Møller M, Arabi YM, Loeb M, Gong M N, Fan E, et al. Surviving Sepsis campaign: Guidelines on the management of critically ill adults with Coronavirus Disease 2019 (COVID-19). Crit Care Med. 2020;48(6):e440-e69. doi:10.1097/CCM.0000000000004363.

8. Barrocas A, Schwartz DB, Hasse JM, Seres DS, Mueller CM. Ethical Framework for Nutrition Support Resource Allocation During Shortages: Lessons From COVID-19. Nutr Clin Pract. 2020;35(4):599-605. doi:10.1002/ncp.10500.

9. Retractionwatch. COVID-19, 2020 [Internet] (Consultado el 25 de Julio 2020) Disponible en: https://retractionwatch. com/retracted-coronavirus-covid-19-papers/

10. Laviano A, Koverech A, Zanetti M. Nutrition support in the time of SARS-CoV-2 (COVID-19). Nutrition. 2020;74:110834. doi:10.1016/j.nut.2020.110834.

11. Yu PJ, Cassiere H, DeRosa S, Bocchieri K, Yar S, Hartman A. Hypermetabolism and Coronavirus Disease 2019 [published online ahead of print, 2020 Jun 19]. JPEN J Parenter Enteral Nutr. 2020;10.1002/jpen.1948. doi:10.1002/jpen.1948.

12. Correia MITD. Nutrition in times of Covid-19, how to trust the deluge of scientific information. Curr Opin Clin Nutr Metab Care. 2020;23(4):288-93. doi:10.1097/ MCO.0000000000000666. 